\title{
The ATLAS Silicon Microstrip Tracker - Operation and Performance
}

\author{
Petra Haefner; on behalf of the ATLAS Collaboration \\ Max-Planck-Institut für Physik, \\ München, Germany \\ E-mail: Petra.Haefner@cern.ch
}

\begin{abstract}
In December 2009 the ATLAS experiment at the CERN Large Hadron Collider (LHC) recorded the first proton-proton collisions at a centre-of-mass energy of $900 \mathrm{GeV}$. This was followed by collisions at the unprecedented energy of $7 \mathrm{TeV}$ in March 2010. The SemiConductor Tracker (SCT) is a precision tracking device in ATLAS made up from silicon microstrip detectors processed in the planar p-in-n technology. The signal from the strips is processed in the front-end ASICs working in binary readout mode. Data is transferred to the off-detector readout electronics via optical fibers. The completed SCT has been installed inside the ATLAS experiment. Since then the detector was operated for two years under realistic conditions. Calibration data has been taken and analysed to determine the performance of the system. In addition, extensive commissioning with cosmic ray events has been performed both with and without magnetic field. The sensor behaviour in magnetic field was studied by measurements of the Lorentz angle. After this commissioning phase it arrived to the first LHC pp collision runs in very good shape: $99 \%$ of the SCT strips are operational, noise occupancy and hit efficiency exceed the design specifications, the alignment is already close enough to the ideal one to allow online track reconstruction and invariant mass determination.
\end{abstract}

KEYWORDS: SCT; Microstrip Tracker; Performance.

${ }^{*}$ Corresponding author. 


\section{Contents}

1. Introduction 1

2. Detector Setup 1

3. Detector Operation 2

4. Detector Geometry Monitoring 2

5. Detector Performance 3

6. Beam Conditions Monitoring 5

$\begin{array}{llr}\text { 7. Conclusions } & 6\end{array}$

\section{Introduction}

The SemiConductor Tracker (SCT) is the silicon microstrip detector of the ATLAS experiment [1]. Its setup will be described in Section 2. Details of SCT operation and its geometry monitoring are given in Sections $3 \& 4$, respectively. SCT properties were studied with different operation parameters. The results of the performance studies will be presented in Section 5. The use of the SCT as LHC beam conditions monitor will be described in Section 6.

\section{Detector Setup}

The SCT is composed of three parts, the barrel and two endcaps (see Figure 1). The barrel consists of four concentric cylinder layers equipped with 2112 silicon modules [2]. The two endcaps consist of 9 disks each, equipped with 1976 modules in total [3]. The SCT detector is $5.4 \mathrm{~m}$ long and covers the radial range of $(30-52) \mathrm{cm}$.

The modules are built from two single-sided sensors, that are glued back-to-back at a stereo angle of $40 \mathrm{mrad}$ to allow the measurement of two-dimensional hit positions. Each sensor has 768 p-type strips on n-type silicon with an $80 \mu \mathrm{m}$ pitch. To allow for the different radii of the endcap disks, five types of endcap sensors were produced. The strip length is about $13 \mathrm{~cm}$ for the barrel modules and between (6-12) $\mathrm{cm}$ in the endcap ones. The sensors are mounted onto a baseboard that constitutes the thermal and mechanical structure of the modules. The readout of the modules is performed by six radiation-hard front-end readout chips per side [4].

The SCT detector is designed to reach a $17 \mu m$ hit resolution in the $r-\phi$ direction and a $580 \mu \mathrm{m}$ hit resolution in the z-direction along the beam pipe. 


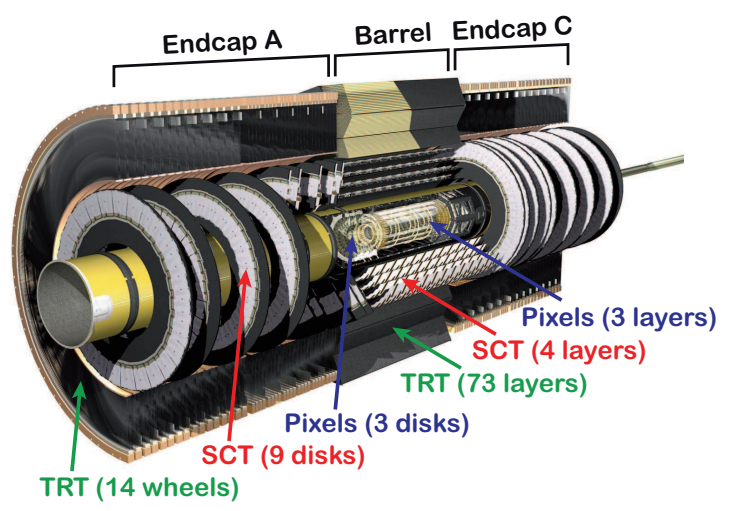

Figure 1. The Inner Detector of the ATLAS experiment consisting of the pixel vertex detector, the silicon microstrip tracker (SCT) and the transition radiation tracker (TRT).

\section{Detector Operation}

During stable proton collisions, the SCT detector is operated at a reverse bias voltage of $150 \mathrm{~V}$. At the beginning of operation full depletion is reached at about $60 \mathrm{~V}$. This will increase with irradiation over the coming years. When LHC beams are non-stable the bias voltage is reduced to $50 \mathrm{~V}$ (standby) to minimize the risk of damaging the silicon modules by possible large charge deposits.

The silicon modules are cooled by an evaporative cooling system based on $C_{3} F_{8}$ [5]. The modules are operated at different temperatures depending on their position in the detector. They are readout in binary mode, which registers whether or not the signal is above a threshold of $1 \mathrm{fC}$. The SCT data acquisition reads out three time bins, where the trigger accept signal lies in the middle bin. A time bin is $25 \mathrm{~ns}$ long corresponding to the LHC bunch spacing.

\section{Detector Geometry Monitoring}

To monitor the short- and long-term stability of the SCT geometry, an optical alignment system is installed in the detector. It is based on Frequency Scanning Interferometry [6] and consists of 842 interferometers that form a geodetic grid of distance measurements between various detector components. As an example, data of 24 out of 144 interferometers that monitor the relative position and orientation of the four carbon-fibre cylinders that support the barrel modules are included in Figure 2. It depicts the movements of the SCT detector for a full cycle of the solenoidal field. As these can be caused both by temperature variations and changes in the magnetic field, the SCT temperature is also shown. It was constant over the time range of the plot. Before the ramp the interferometers measure very stable detector positions with standard deviations of about $11 \mathrm{~nm}$. In contrast, during the solenoid ramp the interferometers detect movements of up to $3 \mu \mathrm{m}$. After the full cycle the position measurements return to the start values to within $49 \mathrm{~nm}$ standard deviations.

Continuous measurements since May 2009 have revealed that the SCT geometry is extremely stable with standard conditions. During non-stable periods that comprise cycles of the solenoidal and toroidal fields, cooling cycles, pixel calibration scans and the SCT winter shutdown the barrel moves typically less than $5 \mu \mathrm{m}$ between barrel layers. 

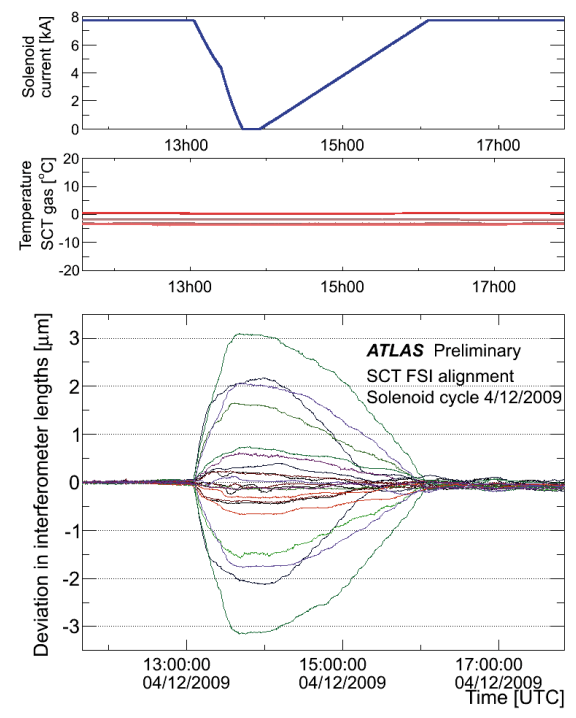

Figure 2. Position measurements of the SCT barrel flanges measured during a run including a full solenoid ramp cycle. Top: solenoid current, Middle: SCT gas temperature, Bottom: interferometer length deviations.

\section{Detector Performance}

In the following, various aspects of SCT performance necessary for good tracking and thus physics results are described.

Timing An important prerequisite for data taking is to have the subdetectors well timed-in with the global ATLAS clock. To achieve that differences in signal travel length and time of flight from the interaction point to the individual SCT modules have to be accounted for. The readout records three time bins centered around the trigger signal (cf. Section 3). Hence, a timed-in detector has a so-called "01X" bin pattern, where we see no hit before the trigger (time bin 0), a hit in correspondence with the trigger signal (bin 1) and have either a hit or no hit after the trigger (bin 2). The optimal timing for all modules would thus correspond to a mean time bin of $\gtrsim 1$. Indeed, the observed mean time bin for the different barrel layers and endcap disks is about 1.2.

Noise Occupancy The noise occupancy of the SCT detector is measured with two methods either with special calibration runs or during normal data taking using random triggers. For calibration, the SCT readout chips are equipped with a charge injection circuit that can apply charges of (016) fC. For a given injected charge, the occupancy vs. threshold is measured. The resulting S-curve is parametrized by a complementary error function. The width of that distribution characterizes the noise. Figure 3 shows an example of such a calibration run. One can see that a typical noise level is around 1500 electrons, whereas the signal threshold corresponds to about 6200 electrons $(1 \mathrm{fC})$. Consequently, the noise occupancy of the SCT detector is very low as shown in Figure 4 using the second method. The noise occupancy is defined as the number of hits observed in random events divided by the number of readout channels. Typical values are on the order of $10^{-5}$. The design specification [7] requires a noise occupancy of $<5 \cdot 10^{-4}$ indicated by the dotted line in Figure 4. Only $0.2 \%$ of all strips of the SCT fail that criterium and consequently are disabled. 


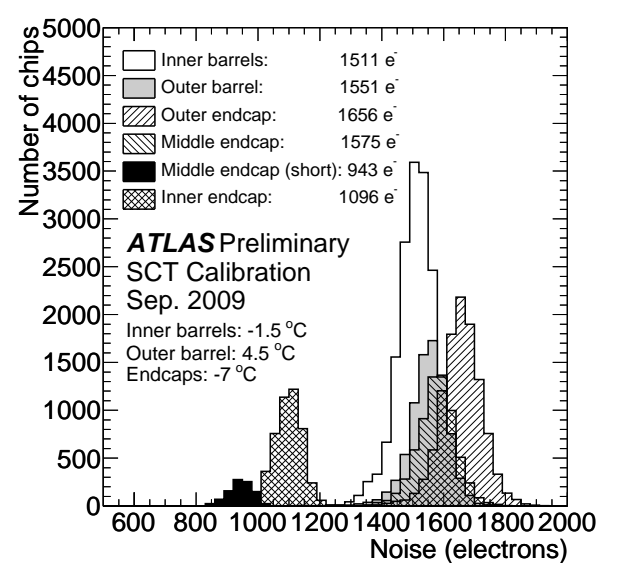

Figure 3. Distribution of input noise values for each chip in the different parts of the SCT. The results are extracted from a calibration run.

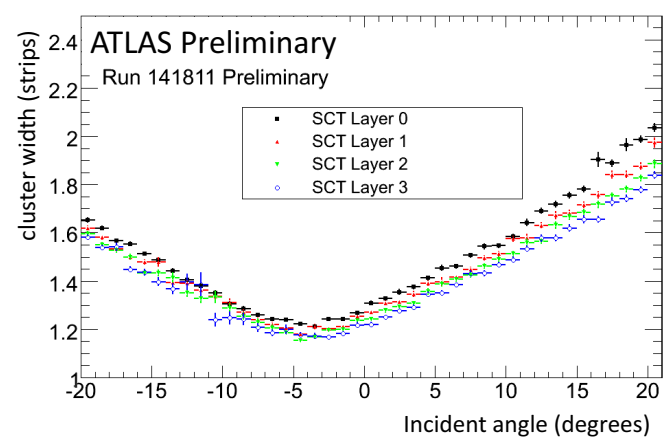

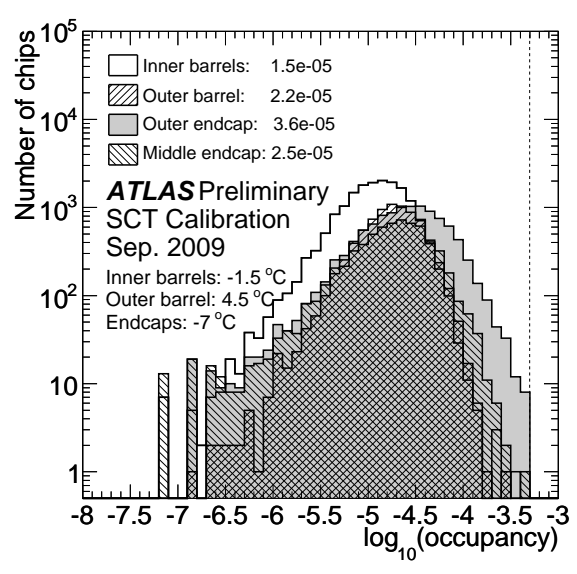

Figure 4. Noise occupancies per chip in the different parts of the SCT. The results are extracted from a cosmic ray run.

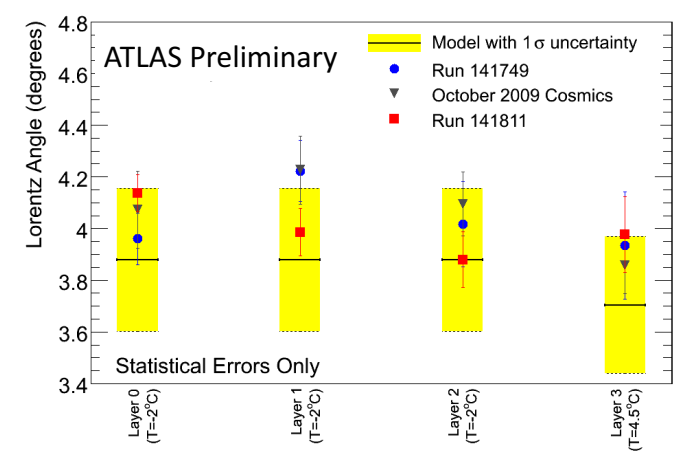

Figure 5. Strip cluster width vs. track incident angle for the four SCT barrel layers (left). The minimum gives the Lorentz angle shown on the right for different run conditions. The model prediction is shown in yellow with a $1 \sigma$ band.

Lorentz Angle Measurements The Lorentz force caused by the $2 \mathrm{~T}$ solenoidal field of the Inner Detector leads to a drift of the charges induced by particles traversing the silicon sensors. The drift angle (Lorentz angle) depends on the magnetic field strength, the module temperature, and the bias voltage. For its determination the strip cluster width is measured vs. the track incidence angle (see Figure 5, left). The track incidence angle which gives the minimal cluster width is the Lorentz angle (cf. Figure 5, right). The temperature dependence is visible in the warmer outermost layer 3, which has to act as thermal shield to the TRT due to a failed heater pad. The measured Lorentz angles are modelled reasonably well.

Hit Efficiency One important factor for the tracking is the hit efficiency defined as the number of hits divided by the number of possible hits. In the latter, dead modules and chips are excluded. For example, the hit efficiency of the SCT for a run with $900 \mathrm{GeV}$ collisions taken in December 2009 and using tracks that have a minimum of 20 TRT hits and 6 SCT hits is $(99.8 \pm 0.1) \%$ on average over all layers / disks. This exceeds the design specification of $99 \%$. 

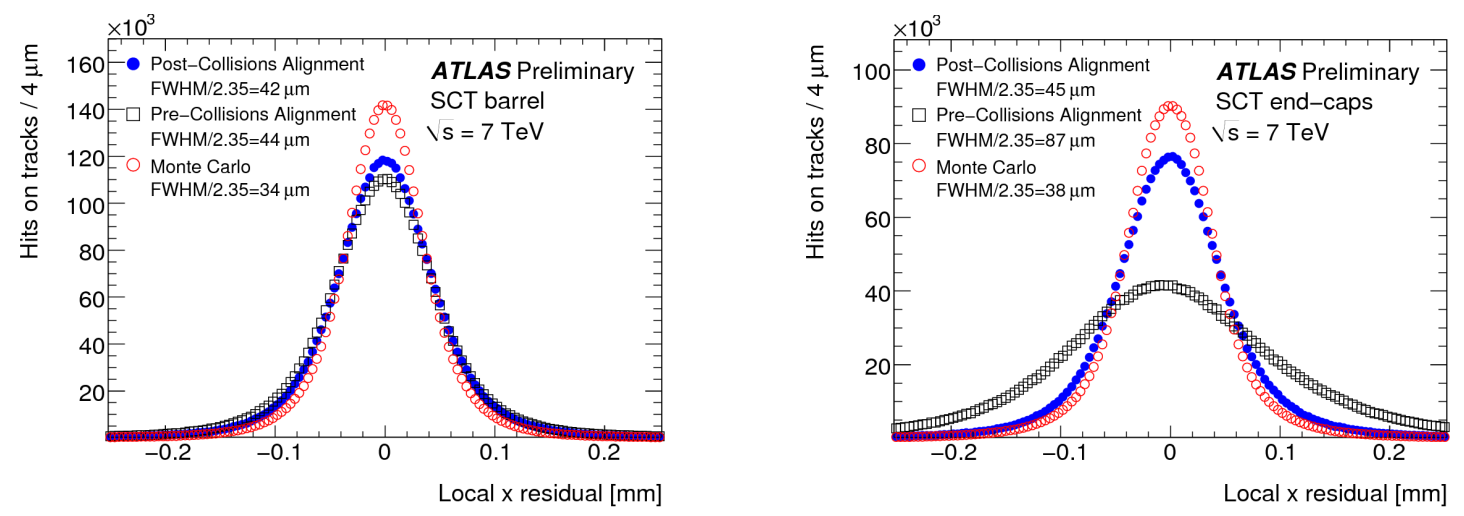

Figure 6. Residual distributions for the barrel (left) and the endcaps (right) [8]. Pre-collision data (cosmics) is shown in black, collision data in blue and simulation in red.

Alignment The alignment with tracks is an essential tool for measuring the "real" geometry of the tracking detectors [8]. It determines disk, layer and module positions and their distortions. The key component is the measurement of the residual defined as the difference between a measured hit position and the expected hit position (on a track). A good alignment is essential for accurate tracking and the derived invariant mass distributions of particles. Due to the geometry of the detector, the barrel could already be very well aligned with cosmic muons traversing the detector perpendicular to the modules. The endcaps can only be aligned well with collision data as cosmic muons traverse them more or less parallel to the modules. This is the reason for the very different quality of the pre-collision alignment shown in Figure 6. However, with collisions the alignment of the endcaps could be easily improved and arrived at the same level as the barrel, which is very close to ideal.

\section{Beam Conditions Monitoring}

Due to its very low noise level and high sensitivity the SCT is an excellent beam conditions monitor. This is especially important during LHC commissioning phases where other silicon precision detectors are switched off for safety. In contrast, the SCT can be run at a reduced voltage at which hit efficiencies of more than $90 \%$ are still achieved and noise levels are only slightly increased (when considering coincidence hits on both module sides). In addition, the SCT is well timed-in (cf. Section 5) and has a very good time resolution. Hence, effects seen in the SCT hit rates can be directly correlated with beam manipulations or losses. As an example, Figure 7, top shows a run including events in which the collimators in front of the Atlas cavern are fully closed and the beam is "dumped" into them (splash events). This results in a large particle shower entering the Atlas cavern. The noise level before and after these events is only a few hits per second, whereas the splashes have five orders of magnitude higher hit rates. Figure 7, bottom shows a long run with collisions. Cosmic showers before beam injection, beam-gas events after injection, and the start and stop of collisions can easily be identified. In contrast, all manipulations of the beam during collisions have very little effect on the hit rates (on a logarithmic scale). This indicates how stable and well-controlled the LHC beam is over a period of many hours. 

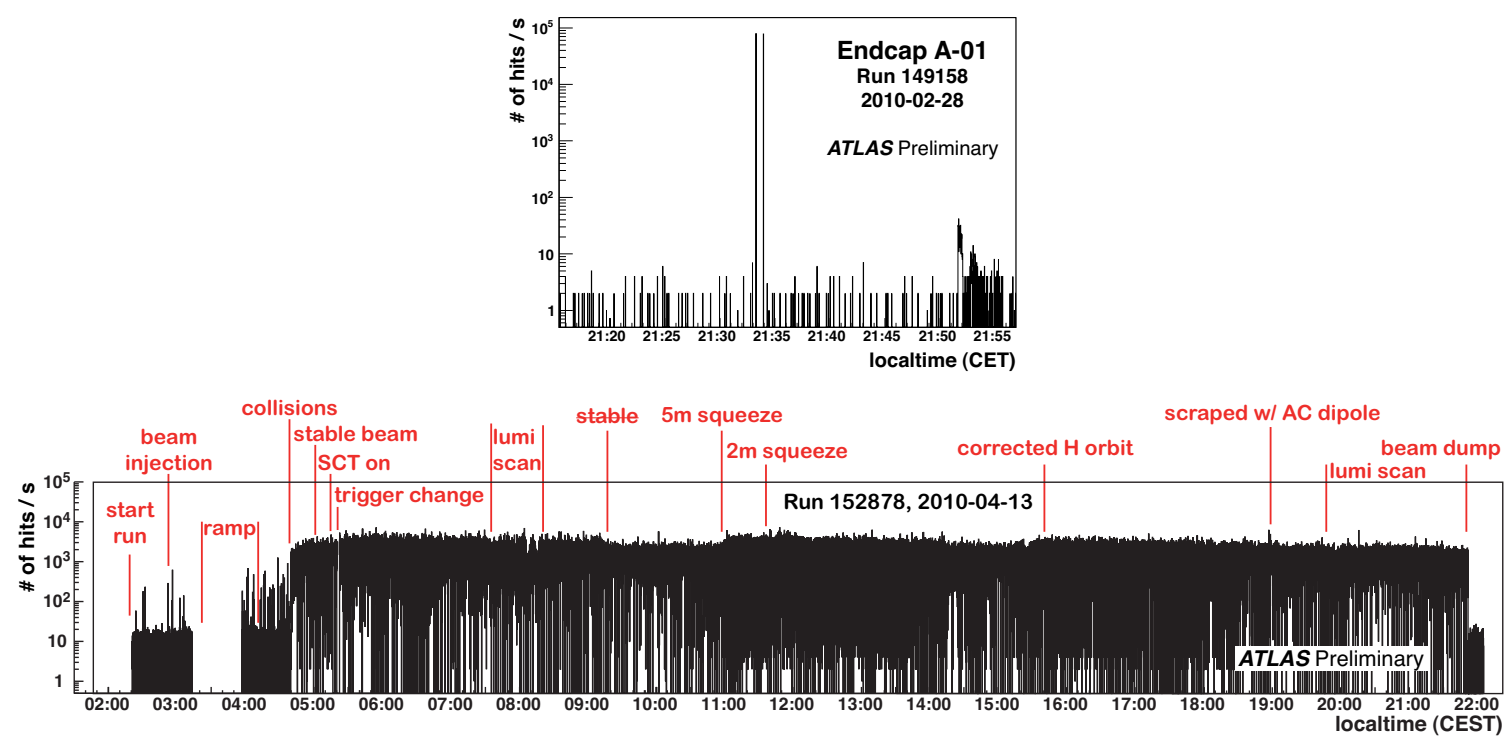

Figure 7. SCT hit rate integrated over a second. Top: A run from Feb. 2010 including two splash events. Bottom: A 20 hour run from April 2010 including $7 \mathrm{TeV}$ collisions.

\section{Conclusions}

The SCT detector is operating stably since its installation in 2008. Its properties and performance have been studied in cosmic ray and proton collision runs. The SCT is a performant, well aligned detector meeting all design specifications. Its low noise level and high sensitivity have made it an important detector for beam conditions monitoring since the beginning of LHC commissioning.

\section{References}

[1] The ATLAS Collaboration, The ATLAS experiment at the CERN Large Hadron Collider, JINST 3 (2008) S08003

[2] A. Abdesselam et al., The barrel modules of the ATLAS semiconductor tracker, Nucl. Instrum. Meth. A 568 (2006) 642

[3] A. Abdesselam et al., The ATLAS semiconductor tracker end-cap module, Nucl. Instrum. Meth. A 575 (2007) 352

[4] F. Campabadal et al., Design and performance of the ABCD3TA ASIC for readout silicon strip in the ATLAS semiconductor tracker, Nucl. Instrum. Meth. A 552 (2005) 561

[5] D. Attree et al., The evaporative cooling system for the ATLAS inner detector, JINST 3 (2008) P07003

[6] S. Gibson et al., Coordinate measurement in 2-D and 3-D geometries using frequency scanning interferometry, Opt. Laser Eng. 44 (2005) 79-95.

[7] Inner Detector Techical Design Report, ATLAS TDR 4, CERN/LHCC/97-16, ISBN 92-9083-102-2

[8] Alignment Performance of the ATLAS Inner Detector Tracking System in $7 \mathrm{TeV}$ proton-proton collisions at the LHC, ATLAS-CONF-2010-067 (2010). 